\title{
Évaluation des pratiques d'hygiène et de prévention de la maladie à coronavirus en milieu hospitalier : Cas des centres d'isolement et de traitement du covid19 au Centre Hospitalo-Universitaire du Point G (CHU-PG) au Mali.
}

\author{
Assessment of hygiene practices and prevention of coronavirus disease in hospitals: Case \\ of isolation and treatment centers for covid19 at the University Hospital of Point $G$ in \\ Mali (CHU-PG).
}

Sanogo $\mathrm{M}^{13^{*}}$, Cissoko $\mathrm{Y}^{2}$, Tonnang RGT ${ }^{1}$, Coulibaly $\mathrm{I}^{1}$,Camara $\mathrm{H}^{4}$, Sacko $\mathrm{A}^{3}$, Diallo $\mathrm{AO}^{5}$, Dao $\mathrm{S}^{2}$.

1. Faculté de Pharmacie de Bamako (FAPH), Université des Sciences, des Techniques, et des Technologies de Bamako (USTTB).

2. Faculté de médecine, d'odontostomatologie (FMOS), Université des Sciences, des Techniques et des Technologies de Bamako (USTTB).

3. Département d'enseignement et de recherche en santé publique (DERSP), Faculté de médecine, d'odontostomatologie (FMOS), Université des Sciences, des Techniques, et des Technologies de Bamako (USTTB).

4. Centre Hospitalier Universitaire Gabriel Touré, service de Cardiologie.

5. Department of Epidemiology, University of North Carolina at Chapel Hill, NC, USA

*Auteur correspondant, Dr Moussa Sanogo, Ph.D, Maitre-Assistant en gestion des systèmes de santé, Email : moussanogo2002@yahoo.fr

\section{Résumé}

But : Ce travail vise à contribuer à l'évaluation des pratiques d'hygiène et de prevention de la maladie à coronavirus covid 19 dans les centres d'isolement et de traitement du Centre Hospitalier Universitaire du Point G (CHU-PG) au Mali. Matériel et méthodes : Il s'agit d'une étude transversale descriptive qui s'est déroulée d'avril à juin 2020. Elle a concerné les centres d'isolement et de traitement de la covid 19. Résultats : Au total 63 agents des deux centres d'isolement et de traitement du covid19 de l'hôpital ont été enquêtés. Il s'agit des médecins spécialistes $(3,2 \%)$, des médecins généralistes $(30,2 \%)$, des médecins en cours de spécialisation (DES) $3,2 \%$, des techniciens supérieurs de santé $3,2 \%$, et des infirmiers $60,3 \%$. Parmi ce personnel, $66,7 \%$ ont suivi une formation sur le lavage des mains et la prévention des infections liées aux soins, tandis que seulement 30,2 l'ont été sur la préparation de la solution hydro alcoolique. Certaines insuffisances ont été constatées dans la pratique du lavage des mains, l'observation des gestes barrières. Par endroit, le déficit dans le port des équipements de protection individuelle et la mauvaise qualité du matériel de nettoyage ont été rappelés. La gestion des déchets demeure problématique du fait de la qualité du personnel et du non-respect des procédures. Conclusion, le respect des mesures d'hygiène et de prevention de la covid 19 pose un problème à l'hôpital du point $\mathrm{G}$ et fait ressortir des manquements liés à la formation, l'utilisation des dispositifs de protection et de prevention, l'organisation des services, la gestion des déchets. Nous recommandons à la direction de l'hôpital, la mise en place d'un service d'hygiène hospitalière doté de compétences et de moyens, le renforcement de la formation du personnel. Le comité d'hygiène et de salubrité doit constituer une sorte de veille des pratiques d'hygiène et de prevention.

Mots clés : Hygiène, prévention, Covid 19, risque, personnel, Hôpital

Summary

Purpose: This work aims to contribute to the evaluation of hygiene practices and prevention of coronavirus disease covid 19 in the isolation and treatment centers of the University Hospital of Point G (CHU-PG) in Mali. Material and methods: This is a descriptive cross-sectional study which took place from April to June 2020. It concerned the isolation and treatment centers for covid 19. Results: A total of 63 agents from the hospital's two isolation and treatment centers for covid19 were surveyed. These are medical specialists (3.2\%), general practitioners $(30.2 \%$ ), doctors in the course of specialization (DES) $3.2 \%$, senior health technicians $3.2 \%$, and nurses $60.3 \%$. Of this staff, $66.7 \%$ received training on hand washing and prevention of healthcare-related infections, while only 30.2 were on the preparation of the hydro-alcoholic solution. Some shortcomings were noted in the practice of hand washing, the observation of barrier gestures. In places, the deficit in the wearing of personal protective equipment and the poor quality of cleaning equipment were recalled. Waste management remains problematic due to the quality of the staff and the non-compliance with procedures. Conclusion, compliance with hygiene and covid 19 prevention measures poses a problem at the G-spot hospital and highlights shortcomings related to training, the use of protection and prevention devices, the organization services, waste management. We recommend that the hospital management set up a hospital hygiene service with skills and resources, and strengthen staff training. The hygiene and sanitation committee must act as a sort of watch on hygiene and prevention practices.

Keywords: Hygiene, prevention, Covid 19, risk, personnel, Hospital 


\section{Introduction}

Le monde fait face depuis décembre 2019 a une nouvelle menace sanitaire dénommée « coronavirus », en abrégée COVID-19 apparu pour la première fois à Wuhan, province du Hubei en CHINE, chez des clients du marché aux poissons, puis s'est étendu à l'échelle nationale, puis internationale (1); le rapport de l'OMS à la date du 22 mars 2020 fait un bilan de 382108 cas confirmés et 18433 décès dans le monde (2). COVID-19 a été déclarée comme pandémie, dans la déclaration de presse du directeur général de l'OMS du 11 mars 2020 (2). On compte actuellement à travers le monde 37808273 cas confirmés, 1080790 décès soit $2,8 \%$ et 1584631 cas en Afrique avec 38598 décès soit un taux de décès $2,4 \%$ (3). Le 7 janvier 2020, la communauté scientifique de Chine l'a décrite comme appartenant à la famille des coronavirus, baptisé temporairement n-2019COV puis définitivement COVID-19 SARS-COV-2 (1). La déclaration du 30 janvier 2020 de l'OMS l'a définie comme une maladie à caractère d'urgence de portée internationale de santé publique à l'épidémie (2). Cao et all (4) précisent au passage l'absence de traitement qui aurait été prouvé efficace à toute maladie sévère causé par l'infection au SARSCOV-2. Face à l'absence de traitement et de vaccin dont l'efficacité pour traiter ou prévenir la COVID19 est avérée, les pays ont pu établir des protocoles standards propres afin de traiter les différents symptômes reposant sur l'état clinique des patients. En vue de tester différents médicaments existant et nouveaux, l'essai clinique Solidarité a été lancé par l'OMS et ses partenaires dans le but de trouver un traitement efficace contre la maladie (4). Pour l'heure, la prévention reste la meilleure stratégie de lutte pour limiter le risque de propagation de la maladie. Une surveillance continue de la situation et des mesures exigées aux pays conformément au règlement sanitaire international (2005) ont été rappelés (2). C'est ainsi que plusieurs mesures ont été préconisées allant du lavage des mains et l'hygiène du milieu, au contrôle des températures, en passant par les mesures de confinement et d'isolement. Le Mali a enregistré son premier cas le 25 mars 2020 (5), compte au 8 janvier 2021, 7512 cas confirmés et 290 décès (soit environ $4 \%$ de taux de décès) (3), et connait une progression exponentielle de cas confirmés. C'est pourquoi le ministère de la santé a décidé de renforcer les mesures de prévention dans le cadre de la COVID19. La situation préoccupante justifie ce choix de recherche afin de comprendre les dispositions prises pour prévenir, stopper et éliminer le virus. Nous allons particulièrement nous intéresser aux méthodes et moyens mobilisés dans le cadre du respect des mesures préconisées pour les gestes barrières, le lavage des mains, le nettoyage et la désinfection des surfaces, le port des masques et autres équipements de protection individuels et collectifs, la prise de température et le rapportage des évènements critiques. Ainsi, nous allons apporter une contribution dans la gestion de cette pandémie du SRAS-COV2 à travers une étude qui passera en revue le niveau de préparation des établissements hospitaliers maliens dans la réponse à la pandémie à COVID-19. Il s'agit de juger les mesures pratiques initiées dans le cadre de l'hygiène et de la prévention, et leur niveau d'application par le personnel soignant de l'hôpital du Point G (HPG).

\section{Méthodologie}

Concrètement, il s'agissait d'une étude descriptive transversale allant d'avril 2020 à juin 2020, soit une période de 3 mois. A cet effet, l'ensemble du personnel de l'Hôpital du Point G affecté au niveau des centres d'isolement et de traitement de la maladie à coronavirus covid 19 a été impliqué. L'intervention a consisté en la collecte d'informations essentielles à partir de la lecture de documents, en échanges avec le personnel soignant impliqué dans la gestion quotidienne de l'épidémie à coronavirus au niveau de l'HPG, en l'observation de certaines pratiques au niveau des services médicaux de l'hôpital.

\section{Type et population d'étude}

Le CHU du Point $\mathrm{G}$ est un établissement public hospitalier qui a mis en place en mars 2020 le premier centre d'isolement et de traitement de la maladie à coronavirus covid 19 affilié au service des maladies infectieuses et tropicales, puis plus tard en juin, un second centre fut créé et rattaché au service de pneumophtisiologie. Notre population d'étude était composée de personnel médical et de soutien travaillant dans les centres d'isolement et de traitement de la covid 19 de l'hôpital du point G. Par souci de triangulation des données, d'autres acteurs ont été impliqués : il s'agit des services médicaux (19 services) qui n'étaient pas directement impliqués dans la prise en charge de la covid 19 mais tenus de respecter les mesures sanitaires et informés des décisions prises.

\section{Echantillonnage}

Nous avons utilisé un échantillonnage nonprobabiliste à proportion raisonnée en ce qui concerne le personnel travaillant au sein des centres d'isolement et de traitement de la covid 19. Un tableau pour chaque groupe des effectifs totaux du personnel affecté dans les centres d'isolement et de traitement de la covid 19 a été adressé. C'est ainsi qu'au niveau des centres d'isolement et de traitement, soixante-trois agents prêt à répondre à nos questions ont été sélectionnés (n $1=63)$ sur un effectif global de cent dix agents $(n=110)$, dix-neuf services médicaux ont également été visités. Le circuit du patient a été déterminé par un algorithme contenu dans les directives nationales de lutte contre de la covid 19, au sein de l'hôpital, un autre algorithme décrivait le circuit interne. 


\section{Collecte et traitement des données}

Pour la collecte des données, nous avons utilisé un questionnaire individuel pour juger de la connaissance générale sur la pandémie à covid 19 et les mesures d'hygiènes et de prevention spécifiques adressées. Une grille d'observation pour évaluer les pratiques du personnel et quantifier les besoins au niveau des centres d'isolement et de traitement, et des services médicaux visités. Elle a été testée sur un petit échantillon puis corrigé et validé avant l'enquête proprement dite. Les procédures opérationnelles établies par les praticiens des deux centres étaient basées sur les directives nationales étaient les normes standards pour l'évaluation. Les fiches d'enquête étaient anonymes et seul le numéro de dossier était utilisé pour la saisie et l'analyse des données. Les données ont été saisies sur Microsoft world 2019 et analysées sur le logiciel SPSS 26. Les données bibliographiques ont été rédigées selon le style Vancouver à l'aide du logiciel ZOTERO.

\section{Variables collectées}

Les données ont été collectées autour de cinq variables indépendantes pour expliquer le niveau de respect des pratiques d'hygiène mesures et de prévention de la covid 19, à savoir: (1) la qualification du personnel soignant des centres covid19, (2) les dispositifs de protection existants contre la covid19, (3) les mécanismes de gestion des déchets biomédicaux, (4) les mesures de réorganisation du travail dans les services au vu du contexte de la covid 19, et enfin (5) les attitudes et pratiques du personnel soignant.

\section{Résultats}

\section{Qualifications du personnel soignant des centres covid19}

Nous avons enquêté soixante-trois (63) personnes qui sont du personnel soignant et de soutien et visité dix-neuf (19) services médicaux.

Tableau I : Répartition du personnel soignant impliqué dans la prise en charge de covid-19 au CHU Point $\mathrm{G}$ selon la qualification au 30 juin 2020

\begin{tabular}{lcccccc}
\hline Personnel & \multicolumn{3}{c}{ Effectif (n) } & \multicolumn{3}{c}{ Pourcentage (\%) } \\
& O & N & Total & O & N & Total \\
\hline Spécialistes & 2 & 4 & 6 & 3,2 & 8,5 & $33 \%$ \\
DES & 2 & 8 & 10 & 3,2 & 17 & $20 \%$ \\
Généralistes & 19 & 9 & 28 & 30,2 & 19 & $68 \%$ \\
TSS & 2 & 26 & 28 & 3,2 & 56 & $7 \%$ \\
TS & 38 & 0 & 38 & 60,3 & 0 & $100 \%$ \\
Total & 63 & 47 & 110 & 100 & 100 & $57 \%$ \\
\hline
\end{tabular}

Globalement, le personnel interviewé représentait plus de $50 \%$ de l'effectif du personnel affecté au traitement des malades de la covid 19 indépendamment de la catégorie. La moyenne d'âge était de 30 ans, les extrêmes étaient de 20 à 48 ans. Les hommes étaient plus représentés que les femmes avec un sex-ratio de 1,86. Le personnel est dominé par les infirmiers soit $60,3 \%$, suivi des médecins généralistes à $30,2 \%$. Il est à déplorer un déficit de spécialistes en maladies infectieuses et tropicales, qui ne représentait que $3,2 \%$ du personnel interviewé dans les centres d'isolement et de traitement de la covid 19.

Le personnel avait une expérience en gestion des maladies infectieuses de façon générale dans 38,1\% des cas, par contre $4,8 \%$ ont affirmé être plutôt impliqués à d'autres activités de soins lors de leur travail dans d'autres établissements publics hospitaliers.

Tableau II : Formations reçues par le personnel soignant dans le cadre de covid19 à l'hôpital du point $\mathrm{G}$ au 30 juin 2020 .

\begin{tabular}{|c|c|c|c|c|}
\hline \multirow[t]{2}{*}{ Formation } & \multicolumn{2}{|c|}{ Oui } & \multicolumn{2}{|c|}{ Non } \\
\hline & $\mathbf{N}$ & $\%$ & $\mathbf{N}$ & $\%$ \\
\hline le port et le retrait d'EPI & 63 & 100 & 0 & 0 \\
\hline $\begin{array}{l}\text { la préparation des solutions } \\
\text { désinfectantes }\end{array}$ & 19 & 30,2 & 44 & 69,8 \\
\hline $\begin{array}{l}\text { la recherche des donnés } \\
\text { littéraire sur la COVID } 19\end{array}$ & 57 & 90,5 & 6 & 9,5 \\
\hline le lavage des mains & 42 & 66,7 & 21 & 33,3 \\
\hline $\begin{array}{l}\text { la prévention de la } \\
\text { COVID-19 }\end{array}$ & 63 & 100 & 0 & 0 \\
\hline $\begin{array}{l}\text { la prévention contre les } \\
\text { infections (PCI) }\end{array}$ & 42 & 66,7 & 21 & 33,3 \\
\hline $\begin{array}{l}\text { Aviez-vous été formé au } \\
\text { prélèvement PCR ? }\end{array}$ & 24 & 38,1 & 39 & 61,9 \\
\hline
\end{tabular}

Le personnel a affirmé avoir suivi une formation sur la préparation des solutions désinfectantes dans $30,2 \%$ des cas ; $90,5 \%$ pour le suivi des données littéraires sur la covid 19. Cependant, $66,7 \%$ disent avoir suivi une formation sur le lavage des mains et la prévention contre les infections liées aux soins (PCI). Tout le personnel affecté dans les centres d'isolement et de traitement affirme avoir suivi une formation sur le port et le retrait des équipements de protection notamment les bottes, les gants en vrac, les gants de ménage. Il a été rapporté une discontinuité parfois dans l'approvisionnement en eau potable dans les services.

Tableau III : Répartition des services de l'hôpital du Point $G$ selon la disponibilité des matériels techniques, équipements et consommables d'hygiène et de prévention des risques au 30 juin 2020

\begin{tabular}{lcccc}
\hline \multicolumn{1}{c}{ Variables } & \multicolumn{2}{c}{ Effectif (n) } & \multicolumn{3}{c}{ Pourcentage (\%) } \\
& oui & Non & oui & non \\
\hline Thermoflash & 3 & 16 & 15,8 & 84,2 \\
Usage EPI & 2 & 17 & 10,5 & 89,5 \\
Matériel & 6 & 13 & 31,6 & 68,4 \\
Masque et gel & 3 & 16 & 15,8 & 84,2 \\
aux malades & & & & \\
Kit protectif & 4 & 15 & 21,1 & 78,9 \\
$\begin{array}{l}\text { Disponibilité de SHA } \\
\text { Toujours }\end{array}$ & 8 & 11 & 42,1 & 57,9 \\
Parfois & 11 & 8 & 57,9 & 42,1 \\
\hline
\end{tabular}

L'hôpital a mis en place une unité de production en oxygène (extracteur d'oxygène) censé approvisionner les centres d'isolement et de traitement et développer une production locale du 
gel hydro alcoolique au niveau de la pharmacie hospitalière. Le personnel supplémentaire a été déployé au niveau des centres d'isolement et de traitement covid19 pour renforcer les équipes existantes. Le centre de tri aménagé dans le cadre de la riposte Ebola a été renforcé et le personnel formé au lavage des mains et à l'utilisation des thermomètres (Thermo flash).

Les services, dans $21 \%$ des cas ont reçu des dispositifs de prévention des infections composés de kits de lavage, savons, bavettes, masques etc. fournis par des donateurs privés et particuliers. Dans trois (3) services (soit 3/19, 15,8\% des cas), les malades ont reçu des masques et du gel hydro alcoolique. Le matériel portatif de contrôle de la température (les thermoflah) était disponible dans trois services (soit 3/19, 15,8\% des cas). Par ailleurs, la solution hydro alcoolique n'était régulièrement disponible que dans huit services (8/19, soit $42,1 \%$ des cas) tandis que $57,9 \%$ des services n'en disposaient que de façon occasionnelle.

Lors de notre visite des locaux, nous avons remarqué que des dispositions particulières ont été prises au niveau de la morgue de l'hôpital en identifiant dans la chambre froide des emplacements spécifiques pour les malades covid19 décédés, en fournissant de l'équipement de protection individuelle au personnel et au service de la buanderie en dotant régulièrement ce dernier de bottes, de gants, de bavettes, de tabliers, de fessières et de bonnets, et la solution chlorée. Le service d'hygiène disposait de chariots spécifiques pour le transport des malades décédés et des déchets liés aux activités des centres covid19. Les frigos réservés par l'accueil des décès liés au covid19 étaient pulvérisés après chaque libération d'un cadavre.

\section{Mécanismes de gestion des déchets biomédicaux solides}

Tableau IV : Mécanismes de gestion des déchets biomédicaux solides issus des services de de l'hôpital du point G à la date du 30 juin 2020 .

\begin{tabular}{lcccccc}
\hline Mécanisme & \multicolumn{3}{c}{ Effectifs (n) } & \multicolumn{3}{c}{ Pourcentage\% } \\
de gestion & $\mathrm{Ou}$ & $\mathrm{No}$ & $\mathrm{NSP}$ & Oui & No & NS \\
des déchets & $\mathrm{i}$ & $\mathrm{n}$ & & & $\mathrm{n}$ & $\mathrm{P}$ \\
\hline Incinération & 19 & 0 & 0 & 100 & 0 & 0 \\
Enfouissment & 0 & 0 & 19 & 0 & 0 & 100 \\
Recyclage & 0 & 19 & 0 & 0 & 100 & 0 \\
Tri & 0 & 19 & 0 & 0 & 100 & 0 \\
Pulvérisation & 11 & 8 & & 58,9 & 42,1 & \\
Emballage & 19 & 0 & 0 & 100 & 0 & 0
\end{tabular}

sécurisée

\section{NSP : Ne sais pas}

Les déchets liquides ne faisaient pas l'objet d'une gestion particulière, ils étaient déversés dans les égouts ou les lavabos suivant le même mécanisme d'acheminement des eaux usées ordinaires via la station de traitement des eaux usées de l'hôpital du point $\mathrm{G}$.
Tableau V : La fréquence de nettoyage, de pulvérisation et d'évacuation des déchets au niveau des services de l'hôpital du point $\mathrm{G}$ à la date du 30 juin 2020.

\begin{tabular}{|c|c|c|c|c|}
\hline \multirow[t]{2}{*}{ Fréquence } & \multicolumn{2}{|c|}{ Effectifs(n) } & \multicolumn{2}{|c|}{ Pourcentage \% } \\
\hline & $\begin{array}{c}\text { Toute } \\
\text { les } \\
12 \mathrm{H}\end{array}$ & $\begin{array}{c}\text { Toute } \\
\text { les } \\
24 \mathrm{H}\end{array}$ & $\begin{array}{c}\text { Toute } \\
\text { les } \\
12 \mathrm{H}\end{array}$ & $\begin{array}{c}\text { Toute } \\
\text { les } \\
24 \mathrm{H}\end{array}$ \\
\hline $\begin{array}{l}\text { Nettoyage des } \\
\text { locaux }\end{array}$ & 19 & 0 & 100 & 0 \\
\hline $\begin{array}{l}\text { pulvérisation } \\
\text { des locaux }\end{array}$ & 8 & 11 & 42,1 & 57,9 \\
\hline $\begin{array}{l}\text { Stockage des } \\
\text { déchets }\end{array}$ & 5 & 14 & 26,3 & 73,7 \\
\hline
\end{tabular}

La durée de stockage des déchets solides était de 12 heures selon $26 \%$ des cas et de 24 heures donc une journée entière pour environ $74 \%$ du personnel. Le tri à la production n'était pas une pratique généralisée, dans les centres d'isolement et de traitement on pouvait parfois observer que les déchets piquants et coupants n'étaient pas séparés des ordures ménagères. Dans tous les services, l'emballage plastique (des sceaux parfois) était utilisé pour le stockage temporaire des déchets, suivi de leur incinération. Les difficultés liées à l'évacuation des déchets ont été rappelées. Ce transport se fait du lieu de production (dans les centres de traitement covid19) au lieu de traitement ou sont implantés les incinérateurs. Le pré traitement se faisait dans les services et les centres de traitement par le moyen de pulvérisation de solution chloré dans 11/19 des services (soit 58,9\% des cas) sur les déchets avant leur incinération au niveau des incinérateurs de l'hôpital.

\section{Mesures de réorganisation prises dans les services le cadre de la COVID 19}

Selon les services, les mesures préconisées étaient appliquées différemment. Le contexte de la covid 19 oblige de nouvelles options de travail notamment le télétravail et les réunions en ligne. Dans notre échantillon, seulement un service sur dix-neuf effectuait des staffs en ligne (soit 5,3 des cas) pendant que quatorze (14) services continuent le staff en mode présentiel avec réduction de l'effectif présent (soit $73,70 \%$ des cas).

Certaines affiches avec des messages de sensibilisation à la covid 19 ont été mises en place dans les salles d'attente de deux (2) services sur dix-neuf (19) soit 10,5\% des cas, la sensibilisation verbale des malades était pratiquée dans 52,6\% des services. Dans trois (3) services, les salles ont été aménagées de telle sorte à respecter la distanciation physique entre les malades, pendant qu'un seul service disposait de salle d'isolement en cas de cas suspect. Il est regrettable de constater que la prise de température systématique n'était appliquée que dans trois (3) services sur dix-neuf (19) soit 15,8\% des cas. Enfin, la gestion des accompagnants des malades demeure un problème majeur, cela 
contribue à grossir le nombre de personne dans les salles d'hospitalisation et les couloirs des services. Le nombre d'accompagnant par malade a diminué dans sept (7) services sur dix-neuf soit $36,8 \%$ des cas, par contre l'effectif pléthorique continu à se vivre dans les autres services indépendamment du contexte actuel de contagion lié à la COVID 19.

Tableau VI : Mesures de réorganisation prises dans le cadre de la covid 19 au niveau des services de l'hôpital du point $\mathrm{G}$ au 30 juin 2020.

\begin{tabular}{|c|c|c|c|c|c|c|}
\hline \multirow[t]{2}{*}{ Variables } & \multicolumn{3}{|c|}{ Effectifs } & \multicolumn{3}{|c|}{ Pourcentage(\%) } \\
\hline & $\mathrm{O}$ & $\mathrm{N}$ & NSP & $\mathrm{O}$ & $\mathrm{N}$ & NSP \\
\hline $\begin{array}{l}\text { Réduction } \\
\text { heure de } \\
\text { travail }\end{array}$ & 5 & 14 & 0 & 26,3 & 73,7 & 0 \\
\hline $\begin{array}{l}\text { Réduction } \\
\text { nombre } \\
\text { d'accompag } \\
\text { nant par } \\
\text { malade }\end{array}$ & 7 & 12 & 0 & $\begin{array}{c}36 \\
8\end{array}$ & $\begin{array}{c}63 \\
2\end{array}$ & 0 \\
\hline $\begin{array}{l}\text { Augmentati } \\
\text { on du } \\
\text { personnel }\end{array}$ & 3 & 16 & 0 & $\begin{array}{c}15 \\
8\end{array}$ & $\begin{array}{c}84 \\
2\end{array}$ & 0 \\
\hline $\begin{array}{l}\text { Réorganisati } \\
\text { on du } \\
\text { personnel }\end{array}$ & 6 & 13 & 0 & $\begin{array}{c}31 \\
6\end{array}$ & $\begin{array}{c}68 \\
4\end{array}$ & 0 \\
\hline $\begin{array}{l}\text { Arrêt du } \\
\text { staff }\end{array}$ & 5 & 14 & 0 & $\begin{array}{c}26 \\
3\end{array}$ & $\begin{array}{c}73 \\
7\end{array}$ & 0 \\
\hline $\begin{array}{l}\text { Staff } \\
\text { électronique }\end{array}$ & 1 & 18 & 0 & 5,3 & $\begin{array}{c}94 \\
7\end{array}$ & 0 \\
\hline $\begin{array}{l}\text { Staff avec } \\
\text { réduction } \\
\text { du } \\
\text { personnel }\end{array}$ & 5 & 14 & 0 & $\begin{array}{c}26 \\
3\end{array}$ & $\begin{array}{c}73 \\
7\end{array}$ & 0 \\
\hline $\begin{array}{l}\text { Fiches } \\
\text { signalétique } \\
\text { S }\end{array}$ & 2 & 17 & 0 & $\begin{array}{c}10 \\
5\end{array}$ & $\begin{array}{c}89 \\
5\end{array}$ & 0 \\
\hline $\begin{array}{l}\text { Disposition } \\
\text { de salle pour } \\
\text { cas suspect }\end{array}$ & 1 & 18 & 0 & 5,3 & $\begin{array}{c}94 \\
7\end{array}$ & 0 \\
\hline $\begin{array}{l}\text { Sensibilisati } \\
\text { on des } \\
\text { malades }\end{array}$ & 10 & 9 & 0 & $\begin{array}{c}52 \\
6\end{array}$ & $\begin{array}{c}47 \\
4\end{array}$ & 0 \\
\hline $\begin{array}{l}\text { Prise de } \\
\text { température }\end{array}$ & 3 & 16 & 0 & $\begin{array}{c}15 \\
8\end{array}$ & $\begin{array}{c}84, \\
2\end{array}$ & 0 \\
\hline $\begin{array}{l}\text { Pulvérisatio } \\
\text { n service }\end{array}$ & 4 & 15 & 0 & $\begin{array}{c}21, \\
1\end{array}$ & $\begin{array}{c}78 \\
9\end{array}$ & 0 \\
\hline $\begin{array}{l}\text { Amélioratio } \\
\mathrm{n} \text { de } \\
\text { l'hygiène du } \\
\text { service }\end{array}$ & 4 & 15 & 0 & $\begin{array}{c}21, \\
1\end{array}$ & $\begin{array}{c}78 \\
9\end{array}$ & 0 \\
\hline $\begin{array}{l}\text { Formation } \\
\text { du } \\
\text { personnel }\end{array}$ & 18 & 1 & 0 & $\begin{array}{c}94 \\
7\end{array}$ & 5,3 & 0 \\
\hline $\begin{array}{l}\text { Réorganisati } \\
\text { on des } \\
\text { chaises pour } \\
\text { appliquer la } \\
\text { distanciation }\end{array}$ & 3 & 16 & 0 & $\begin{array}{c}15 \\
8\end{array}$ & $\begin{array}{c}84 \\
2\end{array}$ & 0 \\
\hline
\end{tabular}

Connaissances et attitudes du personnel face à la maladie à coronavirus COVID 19

Tableau VII : Connaissance et attitudes du personnel soignant des centres de prise en charge COVID-19 de l'hôpital du point G sur le mode de transmission, porte d'entrée, contagiosité de la covid 19 , et la conduite à tenir en cas de suspicion face à la maladie à coronavirus covid 19 au 30 juin 2020.

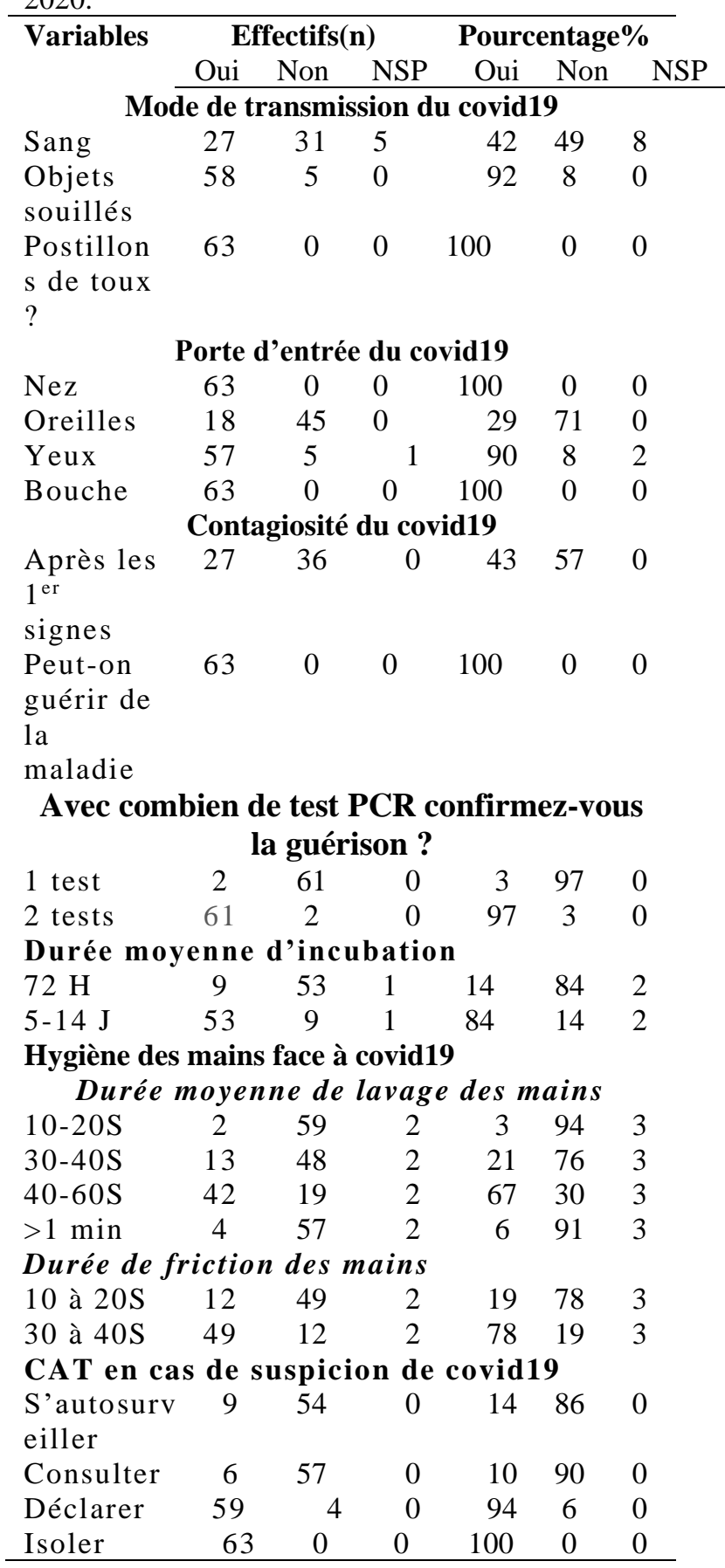

Tous le personnel affirme que les portillons de toux étaient le principal mode de transmission et a cité le nez et la bouche comme les principales voies d'entrée du virus dans l'organisme. Pour $66,7 \%$ des enquêtés, la durée moyenne de lavage des mains était de 40 à 60 secondes, et le pratiquaient 
suivant 6 à 8 étapes comme indiqué par le protocole de lavage des mains selon l'OMS $(6 ; 7)$. La durée moyenne de friction des mains à la solution hydro alcoolique était de 30 à 40 secondes pour $77,8 \%$ des enquêtés suivant 6 à 8 étapes. Ce qui est légèrement supérieure à la norme de l'OMS qui indique tout au plus 20 à 30 secondes suivant 6 à 8 étapes. Le personnel dans sa majorité dispose d'une bonne connaissance sur le mode de transmission du virus de la covid 19.

\section{Discussion}

La discussion sera menée autour de cinq variables indépendantes pour expliquer le niveau de respect des pratiques d'hygiène et des mesures de prévention de la covid 19, à savoir: (1) la qualification du personnel soignant des centres covid19, (2) les dispositifs de protection existants contre le covid19, (3) les mécanismes de gestion des déchets biomédicaux, (4) les mesures de réorganisation du travail dans les services au vu du contexte de la covid 19, et enfin (5) les attitudes et pratiques du personnel soignant.

\section{Formations reçues par le personnel selon la qualification}

Parmi le personnel de santé, les hommes étaient plus représentés avec un sexe ratio de $1,86 \%$ dominé par les infirmiers soit $60,3 \%$. Parmi le personnel, seulement $38,1 \%$ ont déclaré disposer d'une expérience en gestion des maladies infectieuses et $66,7 \%$ l'ont fait sur la prévention des infections notamment le lavage des mains au savon et la friction hydro alcoolique sans une précision sur la méthode adoptée. Ce qui est une obligation de l'OMS dans le cadre de la prévention des infections associées aux soins de la covid 19 tel que consacré dans son guide d'hygiène, qui conseille de tenir compte du temps d'action et des 8 étapes à suivre (7). Aucun participant à l'étude n'a déclaré avoir suivi une visite médicale d'embauche. La visite dans les centres d'isolement et de traitement a permis de constater que le personnel disposait des EPI, cependant des ruptures en gants stériles, surblouses, tabliers et les masques FFP3 ont été rapportées. Ce résultat est supérieur à celui de Helali Amal et al (8) en 2020 qui était de 71,1\% pour le personnel équipé en EPI. Le rythme d'utilisation des EPI était à « usage unique», excepté les lunettes de protection, les visières et les bottes étanches.

\section{Dispositifs de protection et de prévention}

Notre étude a révélé une bonne maitrise de la pratique de l'hygiène des mains à l'eau et au savon et la friction hydro alcoolique par la majorité du personnel respectivement de $66,7 \%$ et $77,8 \%$. Ce résultat est inférieur à celui de Koumedjina Victor (9) en 2019 qui a trouvé que $92,7 \%$ du personnel hospitalier réalisaient le lavage des mains et $80,5 \%$ la friction des mains à la solution hydro alcoolique. Cette différence peut s'expliquer par le peu de formation dispensé jusque-là sur le lavage des mains à l'hôpital du point $\mathrm{G}$. La conduite à tenir devant une suspicion de covid19 rapporté par l'ensemble du personnel était de s'isoler en cas d'exposition accidentelle au virus, suivi de la déclaration pour $93,7 \%$, ce résultat est supérieur à celui de Kansaye (10) qui s'élève à $66,5 \%$. Les affiches de consigne en cas d'exposition accidentelle étaient affichées sur les murs.

Les masques étaient disponibles dans quatre (4) services (soit 15,8\% des cas), cependant un même masque pouvait être porté sur plusieurs jours, ce qui pourrait augmenter le risque d'exposition aux pathogènes comme le déconseille l'OMS $(6 ; 11)$. Seulement, deux services (services de pneumo physiologie et des maladies infectieuses) disposaient d'EPI (soit 10,5\% des cas), le port de ce dernier était systématique. Ces services hébergeaient les deux centres d'isolement et de traitement de la covid 19 à l'hôpital du Point G. Les équipements de protection individuels (ÉPI) représentent le dernier maillon d'une approche graduée qui est en fait la mesure là moins efficace dans la gestion d'un risque. Ce constat est d'autant plus vrai actuellement face à la lutte contre la maladie à coronavirus covid 19 (12).

Même si l'emploi d'EPI est la mesure la plus visible pour éviter la propagation de l'infection, il ne s'agit que de l'une des mesures de lutte contre l'infection et il ne faut pas y avoir recours comme stratégie de prévention principale. En l'absence de mesures de contrôle administratif et technique, les EPI ne présentent que peu d'avantages, comme cela est exposé dans les publications de l'OMS $(6 ; 11)$. La technique de nettoyage du sol pratiqué par les techniciens de surface était celle des sceaux dilués aux désinfectants et pratiqué deux fois par jour (matin et soir) dans l'ensemble des services pendant que la pulvérisation des locaux dans les services était effectuée deux fois par jour dans $57,9 \%$ cas et une fois par jour dans $42,1 \%$ des cas. Cette situation contraste avec les exigences de l'OMS (13). Dans les espaces intérieurs, l'application systématique de désinfectants sur les surfaces environnementales par pulvérisation ou nébulisation (également appelée fumigation ou brumisation) n'est pas recommandée pour combattre la COVID-19. Une étude a montré que la pulvérisation comme mode de désinfection primaire est inefficace pour éliminer les contaminants en dehors des emplacements directement visés. Elle peut au demeurant présenter des risques pour les yeux et provoquer des irritations respiratoires ou cutanées ayant des effets néfastes sur la santé. La pulvérisation ou la nébulisation de certains produits chimiques, tels que le formaldéhyde, les agents à base de chlore ou les composés d'ammonium quaternaire, n'est pas recommandée en raison des effets nocifs qu'elle peut avoir sur la santé du personnel des établissements qui la pratiquent. La 
pulvérisation de désinfectants sur les surfaces environnementales en milieu hospitalier et ailleurs, notamment les foyers des patients, ne sont pas toujours efficaces pour éliminer les matières organiques et n'atteint pas forcément les surfaces couvertes par des objets, les plis dans des étoffes, ou les surfaces ornées de motifs complexes. Lorsqu'un désinfectant doit être appliqué, il convient de le faire au moyen d'un chiffon ou d'une lingette imbibée de produit $(13 ; 12 ; 14 ; 15 ; 16)$.

Mesures de réorganisation des services et de distanciation physique

De façon générale, les services faisaient de timides efforts pour respecter les gestes barrières, ce qui devrait contribuer à diminuer les risques d'exposition à la covid 19 comme préconisé par l'OMS et MSF $(15 ; 17)$. Pour certains, cela est passé par la réduction du nombre d'accompagnant des malades dans 5/19 services, soit 36,8\% des cas, l'arrêt des staffs en mode présentiel classique dans $5 / 19$ services soit $26,3 \%$ des cas, une nouvelle disposition des salles à travers l'aménagement d'une salle pour les contacts et la nouvelle disposition des chaises dans un service 1/19 soit $5,3 \%$ des services. Ce résultat semble mieux que celui des études de koumedjina et Maamri $\mathrm{H}$ and al $(9 ; 18)$ qui ont trouvé que $100 \%$ et $78,6 \%$ des malades et accompagnants dans un hôpital avaient de mauvaises pratiques de prévention. Le respect de la distanciation physique pour la nouvelle disposition des chaises et autres places assises dans les services, ce résultat est largement inférieur à celui de Mohamed et coll (19) soit 76,8\%. Il faut noter une intensification de la sensibilisation des malades dans 10/19 services soit 52,6\% des cas. L'OMS préconise une sensibilisation plus globale des malades qui fréquentent les établissements de santé en période d'épidémie de covid 19 et de leurs accompagnants au respect des mesures sanitaires exigées entre autre, la distanciation physique d'au moins un mètre et le contrôle régulier des températures $(6 ; 11 ; 15)$. Ce qui n'est appliqué que dans 3 services sur 19 soit seulement $15,8 \%$ des cas. Il a été démontré que la distanciation physique, lorsqu'elle est combinée à une bonne hygiène des mains et à l'étiquette respiratoire peut limiter la propagation de la COVID-19 (20). La production de gels hydro alcoolique a été renforcée à travers une responsabilisation de la pharmacie hospitalière. Cette dernière assure la disponibilité du produit dans $42,1 \%$ des services de l'hôpital en dehors des périodes de rupture en matières premières qui peuvent parfois être longues. Ceci est supérieur à l'étude de Mohamed H et coll (19) qui ont trouvé que la pharmacie d'un hôpital africain ne pouvait approvisionner que $6,3 \%$ des services.

\section{Mécanisme de gestion des déchets biomédicaux}

Par ailleurs, nous avons observé que les déchets issus des soins covid-19 étaient transportés de façon manuelle par des chariots spécifiques covid19, l'incinération était citée comme mode de traitement des déchets et dans tous les services aucun tri n'était réellement appliqué pour les déchets pendant que l'OMS recommande un tri selon la typologie des déchets à savoir les piquants coupants, infectieux, pathologiques et pharmaceutiques (21). Par ailleurs, des difficultés d'enlèvement des déchets au niveau des services ont été rapportés allant de $12 \mathrm{~h}(5 / 19$ services soit $26,3 \%)$ à 24 heures (14/19 soit $73,7 \%$ des cas) avec les nuisances liées. Ce résultat se rapproche de ceux obtenus par More Ndiaye et coll et Sanogo et coll $(22 ; 23)$ en 2007 qui ont trouvé une gestion inappropriée des déchets biomédicaux dans des hôpitaux en Afrique du fait, entre autre du retard dans leur enlèvement entrainant des nuisances et la prolifération de germes pathogènes et infectieux.

Connaissances et attitudes pratiques du personnel (CAP)

Certains personnels ont été formés à la préparation d'une solution désinfectante à base de chlore (soit $30,2 \%$ de l'effectif dédié aux centres), et disent tous procéder au nettoyage des surfaces et à la gestion des déchets biomédicaux. En plus de l'absence d'affiche rappelant les procédures à suivre, il existait un déficit en personnel de nettoyage spécialisé à l'hôpital du point $\mathrm{G}$ et dédié à l'hygiène dans un tel contexte spécifique d'épidémie qui est potentiellement à plus grand risque. Ce qui contrarie avec les recommandations de l'OMS et de MSF qui préconisent plutôt un partage de responsabilité entre les acteurs spécialisés sur différents segments de la riposte contre la pandémie à covid $19(13 ; 17)$.

La formation du personnel de nettoyage devrait être fondée sur les politiques et les modes opératoires normalisés de l'établissement de santé et sur les directives nationales. Elle devrait être structurée, ciblée et dispensée comme il se doit (participative et adaptée au niveau d'instruction du personnel, par exemple), et constituer un élément obligatoire du programme d'initiation offert aux nouvelles recrues. Elle devrait notamment comprendre des instructions sur l'évaluation des risques et assurer l'acquisition de compétences probantes, qu'il s'agisse de la préparation fiable de désinfectants, du nettoyage mécanique et de l'utilisation du matériel, de précautions de base ou axées sur la transmission $(13 ; 17)$. Au cours des sessions de formation, l'OMS recommande pour encourager et renforcer le recours à de bonnes pratiques, dans les établissements de santé et les bâtiments publics, les affiches ou autres supports devraient être apposés bien en vue des agents du personnel de nettoyage, entre autres, de manière à rappeler les directives et les procédures à suivre pour la préparation et l'utilisation des désinfectants (13).

Par ailleurs, le circuit d'approvisionnement en eau de l'hôpital n'était pas stable avec des coupures fréquentes d'eau avec son impact sur le respect des 
mesures d'hygiène physique et celle des locaux et du matériel. L'approvisionnement en eau est un impératif pour la sécurisation des soins et leur continuité. « Il doit exister dans l'État partie, en quantité suffisante, des installations, des biens et des services ainsi que des programmes fonctionnels en matière de santé publique et de soins de santé. La nature précise des installations, des biens et des services dépendra de nombreux facteurs, notamment du niveau de développement de l'État partie. Ces installations, biens et services comprendront toutefois les éléments fondamentaux déterminants de la santé tels que l'eau salubre et potable et des installations d'assainissement appropriées, des hôpitaux, des dispensaires et autres installations fournissant des soins de santé (24).

\section{Conclusion}

Le respect des mesures d'hygiène et de prevention des infections liées aux activités de soins de la covid 19 pose un énorme problème à l'hôpital du point $\mathrm{G}$ et fait ressortir des manquements sur la formation du personnel, l'existence et l'utilisation des dispositifs de protection et de prevention de la pandémie, la réorganisation des services au vu du contexte, les mécanismes de gestion des déchets biomédicaux et enfin les connaissances et attitudes pratiques du personnel sur l'épidémie.

L'importance des risques encourus commande l'engagement des responsables de l'hôpital, du niveau clinique au niveau administratif pour améliorer rapidement la situation. Les moyens mobilisés jusque-là sont insuffisants et peu appliqués donc inefficaces. La pratique de l'hygiène demande une prise de conscience individuelle et collective avec une concertation permanente pour évaluer les actions entreprises et apporter l'accompagnement nécessaire. Le non-respect des mesures d'hygiène et de prevention à la covid 19 expose le personnel et les malades et favorise la contamination. Il faudra donc former suffisamment de personnel au respect des mesures préconisées notamment par les directives nationales, celles de l'OMS, de MSF. La pharmacie de l'hôpital dispose de compétences nécessaires à la production en grande quantité de la solution et du gel Hydra alcoolique à prix modéré à condition de disposer permanemment des matières premières nécessaires à sa fabrication.

\section{En clair, nous recommandons :}

À la direction générale de l'hôpital du point $\mathrm{G}$, la mise en place d'un véritable service d'hygiène au sein de l'hôpital tenu par un personnel bien formé et compétent, un renforcement dans la formation et la sensibilisation de l'ensemble du personnel soignant au respect des mesures sanitaires et sensibiliser les malades et leurs accompagnants à défaut d'interdire cette pratique ; aux commissions statutaires de l'hôpital du point G d'intégrer les enjeux de la pandémie dans leur prise de décision médicale en ce qui concerne la commission médicale d'établissement et la commission des soins infirmiers, d'intensifier les actions de sensibilisation à l'endroit du personnel médical et d'instaurer dans les services des comités de suivi évaluation ; au comité d'hygiène et de salubrité de constituer une sorte de veille et de sentinelle des pratiques d'hygiène et assurer la promotion d'une culture d'hygiène au sein de l'hôpital tout en veillant rigoureusement l'application des mesures rigoureuses et contraignantes; au ministère de la santé, instituer un système d'évaluation de l'application des directives nationales en matière d'hygiène et prevention des risques de façon générale, et d'inscrire les pratiques dans un cadre plus normatif et règlementaire, de doter les hôpitaux de moyens conséquents qui leur permettent de réaliser pleinement leurs missions de soins dans le cadre de la covid 19. Les hôpitaux étant déjà saturés de malades dans la plupart des cas, il faudra créer des structures plus autonomes pour assurer une prise en charge de masse des cas de covid 19.

Déclaration de conflit d'intérêt : Les auteurs ne déclarent aucun conflit d'intérêt.

Remerciements : Nous remercions l'administration et tout le personnel de l'hôpital du Point G du Mali, particulièrement ceux du service des maladies infectieuses et tropicales ayant contribués à l'élaboration de ce modeste travail en répondant sincèrement à nos questions.

\section{References bibliographiques}

1. Qi Y, Du CD, Liu T, Zhao X, Dong C. Experts' conservative judgment and containment of COVID19 in early outbreak. Journal of Chinese Governance. 2 avr. $2020 ; 5(2): 140-59$.

2. Organisation Mondiale de la Santé (OMS) : Chronologie de l'action de l'OMS face à la COVID-19, 2020 [Internet]. [Cité 6 Oct 2020]. Disponible sur: https://www.who.int/fr/newsroom/detail/29-06-2020-covidtimeline

3. Economic Community of West African States (Ecowas): Report Covid19 SitREP, Ecowas, Abuja, 12 October 2020. [Cité 14 October 2020]. Http: // www.wahooas.org

4. Cao B, Wang Y, Wen D, Liu W, Wang J, Fan G, et al. A Trial of Lopinavir-Ritonavir in Adults Hospitalized with Severe Covid-19. N Engl J Med. 7 mai $2020 ; 382(19)$ : 1787-99.

5. Ministère de la santé et des affaires sociales : Plan de réponse à la maladie à coronavirus 2019 covid-19, [Les directive nationales dans le cadre de la réponse au covid19] Mali, Avril 2020. Document cadre du Ministère de la santé du Mali, Disponible sur : https: //www.sante.gov.ml

6. World Health Organization (WHO) : Protocole de surveillance de l'infection à SARS-CoV-2 chez les agents de santé, version1, mai 2020. [Visité le 05/01/2021], Disponible sur https: //www.who.int. 
7.World Health Organization (WHO) : Guide pour l'hygiène des mains, version1, mai 2020. [Visité le 05/01/2021], Disponible sur https: //www.who.int. 8. HELALI A and al: The COVID-19 crisis seen by the Algerian pharmacist community. Algerian journal of Pharmacy. Vol 03. Num 01. (2020) 2602-795X. Disponible sur https://www.researchgate.net/profile/Amal_Helali/p ublication/342338651.

9. Koumedjina KV: Evaluation des mesures de prévention contre les infections nosocomiales dans le service des maladies infectieuses du C.H.U. de Point G. [Internet] [Thesis]. USTTB ; 2019 [cité 25 Oct 2020]. Disponible sur : https://www.bibliosante.ml/handle/123456789/3740 10. Kansaye Y. Perceptions et pratiques de prévention reliées à la maladie à virus Ebola au CHU du point G du Mali. 2015 [cité 25 Oct 2020] ; Disponible sur

https://library.adhl.africa/handle/123456789/10274

11. World Health Organization (WHO) : Utilisation rationnelle des équipements de protection individuelle (EPI) contre la maladie à coronavirus 2019 (COVID-19), Orientations provisoires, Avril 2020. [Visité le 07/01/2021], WHO reference number: WHO-2019-nCov-IPC_PPE_use-2020.3fre, Disponible sur https: //www.who.int.

12. Association paritaire pour la santé et la sécurité du travail, secteur « affaires municipales» (APSAM) : Vêtements de sécurité à haute visibilité (Norme CSA Z96 15 FR), Association Canadienne de Normalisation, Canada, 2016, [Visité le 05/01/2021], Repéré et disponible sur https://www. scc.ca/fr/standardsdb/standards/28566.

13. World Health Organization (WHO) : Nettoyage et désinfection des surfaces environnementales dans le cadre de la COVID-19, Orientations provisoires, Mai 2020. [Visité le 06/01/2021], WHO reference number: WHO-2019-nCoV-Disinfection-2020.1fre, Disponible sur https://apps.who.int/iris/handle/10665/332096. 14. Tremblay $M$ et coll: Décontamination des surfaces et objets souillés de sang ou liquides biologiques adaptée aux intervenants d'urgence Prévention contre les risques biologiques au travail, Association paritaire pour la santé et la sécurité du travail, secteur « affaires municipales » (APSAM Canada), 2016, [Visité le 06/01/2021], Repéré et disponible sur https://www. scc.ca/fr/standardsdb/standards/28566.

15. World Health Organization (WHO): Report of the WHO-China Joint Mission on Coronavirus Disease 2019 (COVID-19) 2020, Geneva. [Consulté le 10 mai 2020], Disponible sur https://www.who.int/docs/default-

source/coronaviruse/who-china-joint-mission-oncovid-19-finalreport.pdf.

16. Rutala, W.A., Weber, D.J: Disinfectants for Use Against SARS-CoV-2 | US EPA. Uses of inorganic hypochlorite (bleach) in health-care facilities,
United States Environmental Protection Agency (US-EPA), 2020. [accessed 6 May 2020], disponible sur https://www.epa.gov/pesticideregistration/list-ndisinfectants-use-against-sars-cov2. Clin. Microbiol. Rev. 10, 597-610.

17. Médecins Sans Frontières (msf.fr) : prise en charge des personnes affectées par la Covid-19, Mali, April 2020 [visité en juin 2020], disponible sur http//www.msf.fr > decouvrir-msf , nosopérations > mali-pr.

18. Maamri H, Ben Ayed H, Ben Hamida M, Ben Jmaa M, Trigui M, Ketata N, et coll. Évaluation des pratiques d'hygiène et de prévention du COVID19 en milieu hospitalier. Médecine et Maladies Infectieuses. 1 sept $2020 ; 50(6$, Supplement $)$ : S103-4.

19. Mohamed H et coll : Moyens de prévention du risque d'exposition au SARS-Cov-2: enquête auprès des pharmaciens d'une ville de l'Est algérien. Archives des Maladies Professionnelles et de l'Environnement. 1 août 2020 ;81(4) :341-7.

20. Public Health Ontario : Maladie à coronavirus 2019 (COVID-19), Distanciation physique, Ontario Canada, avril 2020, [Consulté le 10 mai 2020]. Disponible sur https://www.publichealthontario.ca/$/$ media/documents/ncov/factsheet/factsheet-covid19-guide-physical-distancing.

21. Giroult E: Règles de gestion des déchets hospitaliers (pour les pays en développement). Genève, OMS/UEH/EOS ;1996.12p. [Consulté le 10 mai 2020]. Disponible sur https: //www.who.int. 22.. Ndiaye M, Maniboliot Soumah M : Évaluation de la prévention technique et médicale du risque biologique au sein de l'hôpital général de GrandYoff (HOGGY) de Dakar à l'heure de la maladie Ebola. Archives des Maladies Professionnelles et de l'Environnement. 1 juin 2016 ;77(3) :433-4.

23. Sanogo $M$ et coll : Contribution à la mise en place d'un système de gestion durable des déchets biomédicaux à l'hôpital Gabriel Touré du Mali. Le Pharmacien Hospitalier. Volume 42, Issue 170, Septembre 2007, Pages 143-147, Disponible sur: https: //www.researchgate.net

24. De Albuquerque C: Bonnes pratiques de réalisation des droits à l'eau et à l'assainissement de la Rapporteuse spéciale de l'ONU, Lisbonne, février 2012, ISBN 978-2-7466-4501-1, disponible à l'adresse suivante $: \mathrm{http} / / \mathrm{www}$. ohchr.org/BookonGoodPractices.fr 